\title{
RENAL REPLACEMENT THERAPY IN INTENSIVE CARE UNIT AT A TERTIARY CARE CENTER IN NEPAL
}

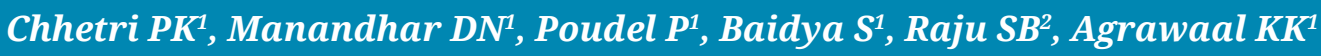

${ }^{1}$ Department of Nephrology, Nepal Medical College Teaching Hospital, Attarkhel, Gokarneshwor-8, Kathmandu, Nepal,

${ }^{2}$ Department of Nephrology, Nizam Institute of Medical Sciences, Hyderabad, India

\begin{abstract}
Acute kidney injury is a major complication in intensive care unit patients. It is associated with increased in-hospital mortality and length of stay. The provision of renal replacement therapy in intensive care is not widely available in resource poor countries like Nepal. The study aims to look into clinical profile and outcome of patients who received renal replacement therapy in intensive care unit. It was an observational study done from 1st October 2016 till 30th September 2017. Patient's demographic data, indications, biochemical tests, outcomes, modality of renal replacement therapy were recorded. Statistical package for the social sciences version 17 was used for statistical analysis. There were total of 649 admissions in intensive care, among which 148 had kidney related complications. Of 148 patients, $69(47 \%)$ received renal replacement therapy. Mean age, urea and creatinine on admission were $50.17 \pm 18.42$ years, $174.54 \pm 63.46 \mathrm{mg} / \mathrm{dl}$ and $8.05 \pm 3.49 \mathrm{mg} /$ dl respectively. They underwent $4.32 \pm 3.09$ sessions and $14.94 \pm 10.88$ hours of renal replacement therapy. Total 42 (61\%) had septic shock on admission and underwent sustained low efficiency dialysis as the modality of renal replacement therapy. In-hospital mortality was 19 (28\%). Presence of septic shock on admission and mean number of ionotropes required $2.05 \pm 1.12$ was statistically significant for in-hospital mortality $(\mathrm{p}=0.01)$. About half of the patients were on mechanical ventilation which was statistically significant for in-hospital mortality $(p<0.001)$. Sustained low efficiency dialysis can be done in patients on ionotropes and patients can be switched over to intermittent hemodialysis.
\end{abstract}

\section{KEYWORDS}

Intensive care unit, Nepal, renal replacement therapy; sustained low efficiency dialysis

\section{CORRESPONDING AUTHOR}

Prof. Pramod Kumar Chhetri

Department of Nephrology,

Nepal Medical College Teaching Hospital, Attarkhel,

Gokarneshwor-8, Kathmandu, Nepal

Email: pkpchhetri@gmail.com 


\section{INTRODUCTION}

Acute Kidney Injury (AKI) has a significant implication in hospital related morbidity and mortality including prolonged hospital stay and mechanical ventilation. ${ }^{1}$ The burden of Chronic kidney disease (CKD) is also escalating and so is end stage renal disease (ESRD). ${ }^{2}$ Patients with CKD have high rates of AKI and vice versa. ${ }^{3}$ Thus, AKI and CKD are two interconnected renal syndromes. ${ }^{4}$ Both these group of patients may land up in intensive care unit (ICU) due to sepsis, pulmonary edema, oliguria, multi organ dysfunction syndrome and may require renal replacement therapy (RRT) which is challenging in them. There are different modalities of RRT available for patients in ICU viz. intermittent peritoneal dialysis (IPD), hemodialysis (HD), hybrid therapy, continuous renal replacement therapy (CRRT). ${ }^{5}$ IPD is less preferred in adults due to its procedural challenge whereas most patients cannot tolerate HD. CRRT has issues with cost and/ or availability. A hybrid therapy called sustained low efficiency dialysis (SLED) can be performed in resource poor setting. Nephrology services were started in Nepal about 30 years ago with the first Nephrology outpatient department in 1984, first HD service in 1988 and first Nephrology unit in 1990 whereas the ICU services were started before Nephrology services in $1973 .{ }^{6}$ There has been significant technological advances in the delivery of RRT, particularly to the critically ill patient population. Despite these advances, critically ill patients continue to carry a poor prognosis. ${ }^{7}$ In a article published by Acharya ${ }^{8}$ on status of ICU services in Nepal, found that RRT was available in the form of HD in only one ICU in Nepal. However, though there were risks involved with transferring patients, HD was done outside ICUs in 14 other hospitals within Kathmandu. Peritoneal dialysis was still the most common form of RRT therapy in ICUs in Kathmandu. There is no update to this data. Hence, this novel beginning of ICU dialysis in the form of SLED was started since January 2012 at Nepal Medical College Teaching Hospital (NMCTH). This study was designed to look into clinical profile, biochemical profile and outcomes of the patients during hospital stay receiving RRT in general ICU at NMCTH.

\section{MATERIALS AND METHODS}

We have followed strengthening the reporting of observational studies in epidemiology (STROBE) guidelines for reporting this hospital based observational study conducted at NMCTH from $1^{\text {st }}$ October 2016 till $30^{\text {th }}$ September 2017. Ethical approval was taken from institutional review committee (NMC-IRC). All the consecutive patients undergoing RRT (SLED or HD) were enrolled in the study after taking informed consent from the patient/ patient's relatives. The primary outcome for the study was all cause in-hospital mortality. The data included demographic of the patients (such as age, sex, address, date of admission), clinical information (relevant brief history, physical examination), biochemical laboratory parameters (serum urea and creatinine), modality of RRT and the outcome of the course. Patients were classified as AKI or AKI on CKD or CKD. The cases were defined based on the definition given by kidney disease improving global outcomes (KDIGO) for AKI and CKD. ${ }^{9,10}$ For statistical analysis SPSS software version 17 was used. Descriptive data and outcomes identified were analyzed using the $\chi 2$ test and independent $t$ test was applied for comparison between mortality

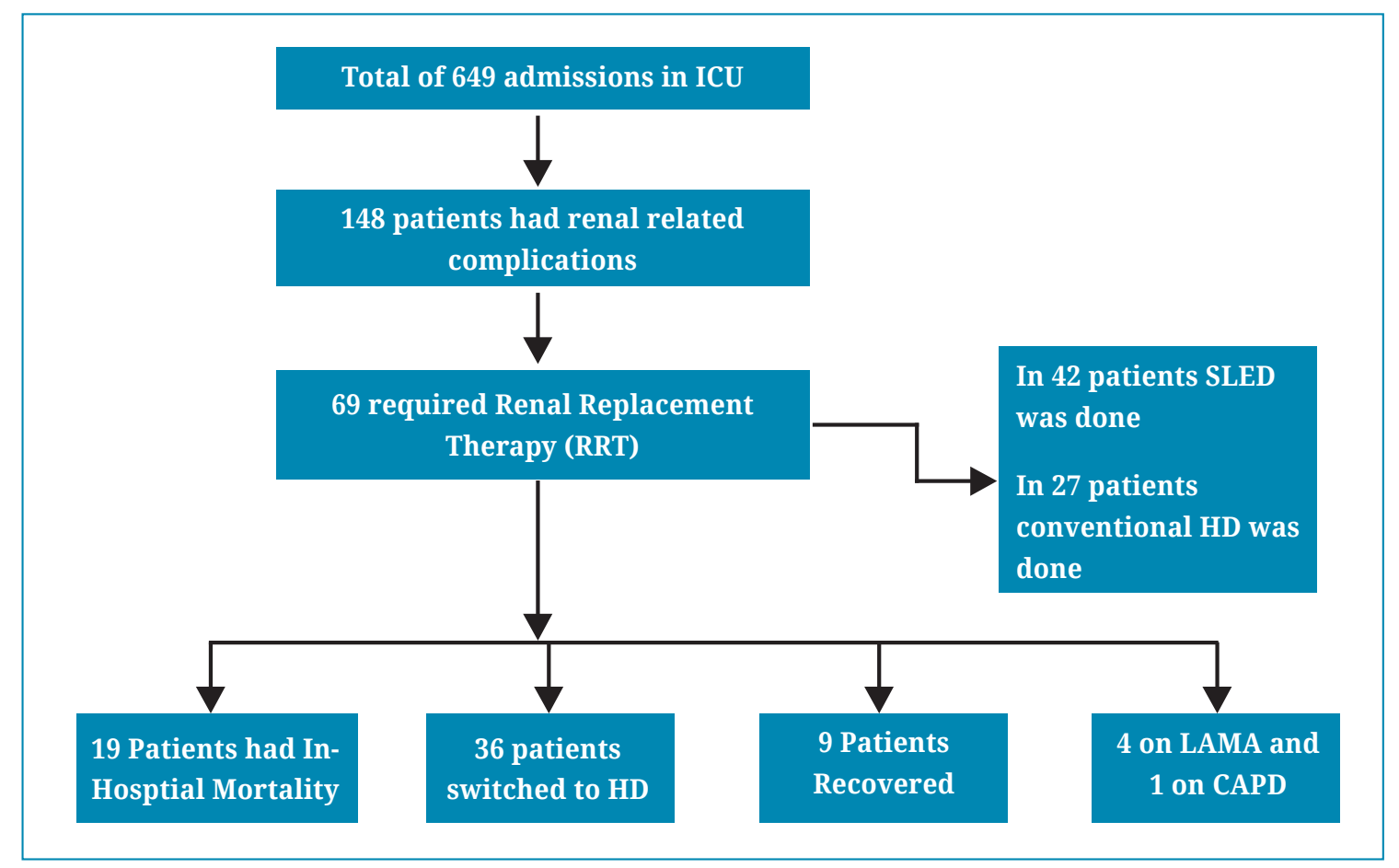

Fig. 1: Flow diagram of study population 
and no mortality groups. The data was presented as percentage or mean \pm standard deviation (SD) or median with inter quartile range (IQR). The confidence interval was $95 \%$ and $\mathrm{p}<0.05$ was used for statistical significance.

\section{RESULTS}

There were 69 patients who underwent RRT in general ICU at NMCTH (Fig. 1) with a male: female ratio of 1:1.09. The baseline characteristic of study population is shown in Table-1. Diabetes mellitus

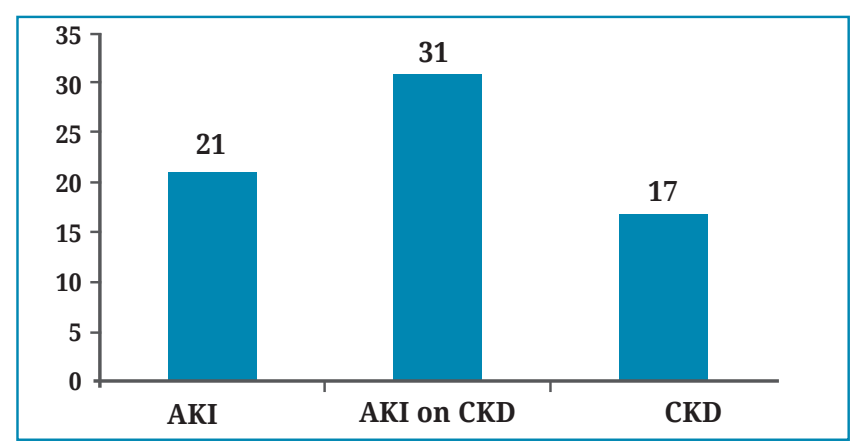

Fig. 2: Number of patients with different diagnosis $(n=69)$ and hypertension were present in 32 (47.0\%) and 42 (61.0\%) patients, respectively.

Total number of patients with AKI and AKI on CKD was 52 (75.4\%) and among them 49/52 (94.2\%) had sepsis as a cause of AKI as shown in Fig. 2. The most common cause of sepsis was pneumonia followed by urosepsis as shown in Fig. 3. The most common pre-existing renal disease in patients requiring RRT was Diabetes (Fig. 4). The most common indication for RRT was refractory metabolic acidosis with oliguria $(n=38,55 \%)$ followed by pulmonary edema $(n=15,28 \%)$. SLED was done in $42(61 \%)$ of total RRT patients among which $31(74 \%)$ were on two or more ionotropes. Among the patients on SLED, 19/42 (45\%) had in-hospital mortality. Among these 19 patients, 10 (53\%) had AKI and nine (47\%) had AKI on CKD. Septic shock was present in $42(61 \%)$. A total of $28(40 \%)$ were on HD prior to admission and $32(46 \%)$ were on mechanical ventilation. The overall in-hospital mortality was seen in $19(28 \%), 36$ (52\%) were switched to HD and 9 (13\%) recovered. Septic shock on admission and need for mechanical ventilation was statistically significant for in-hospital mortality ( $p$ value $<0.001$ ). Bivariate analysis done among mortality and with no mortality is shown in Table-2 and -3 .

\section{Table-1: Baseline characteristics of study population $(n=69)$}

\begin{tabular}{lcccc|} 
Characteristics & Minimum & Maximum & Mean \pm SD & Median (IQR) \\
Age in years & 15 & 89 & $50.17 \pm 18.42$ & $52(34-63)$ \\
Duration of hospital stay in days & 1 & 64 & $12.86 \pm 12.06$ & $8(5-16)$ \\
Serum urea (mg/dl) & 53 & 308 & $174.54 \pm 63.46$ & $175(123-196)$ \\
Serum creatinine (mg/dl) & 3.1 & 18.30 & $8.05 \pm 3.49$ & $7.4(5.4-9.8)$ \\
No. of sessions of RRT & 1 & 16 & $4.32 \pm 3.09$ & $3(2-5)$ \\
Duration of RRT in hours & 2 & 52 & $14.94 \pm 10.88$ & $12(8-18)$ \\
No. of ionotropes & 0 & 4 & $1.23 \pm 1.13$ & $1(0-2)$ \\
\hline
\end{tabular}

\section{Table-2: Comparison of mean parameters with in-hospital mortality (n=69)}

\section{Characteristics}

Age

Serum creatinine $(\mathrm{mg} / \mathrm{dl})$ on admission

Serum urea $(\mathrm{mg} / \mathrm{dl})$ on admission

No. of ionotropes on admission

No. of sessions of RRT on admission

Duration of RRT in hours
Mean \pm SD in patients with mortality $(n=19)$

$$
\begin{gathered}
52.84 \pm 18.59 \\
6.90 \pm 3.05 \\
183.00 \pm 58.60 \\
2.05 \pm 1.12 \\
3.11 \pm 1.85 \\
11.16 \pm 6.20
\end{gathered}
$$

Mean \pm SD in patients with no mortality $(n=50)$

$P$ value

$\begin{array}{cc}49.16 \pm 18.44 & 0.46 \\ 8.49 \pm 3.58 & 0.09 \\ 171.32 \pm 65.48 & 0.49 \\ 0.9 \pm 0.99 & <0.01 \\ 4.78 \pm 3.35 & 0.04 \\ 16.38 \pm 11.94 & 0.07\end{array}$




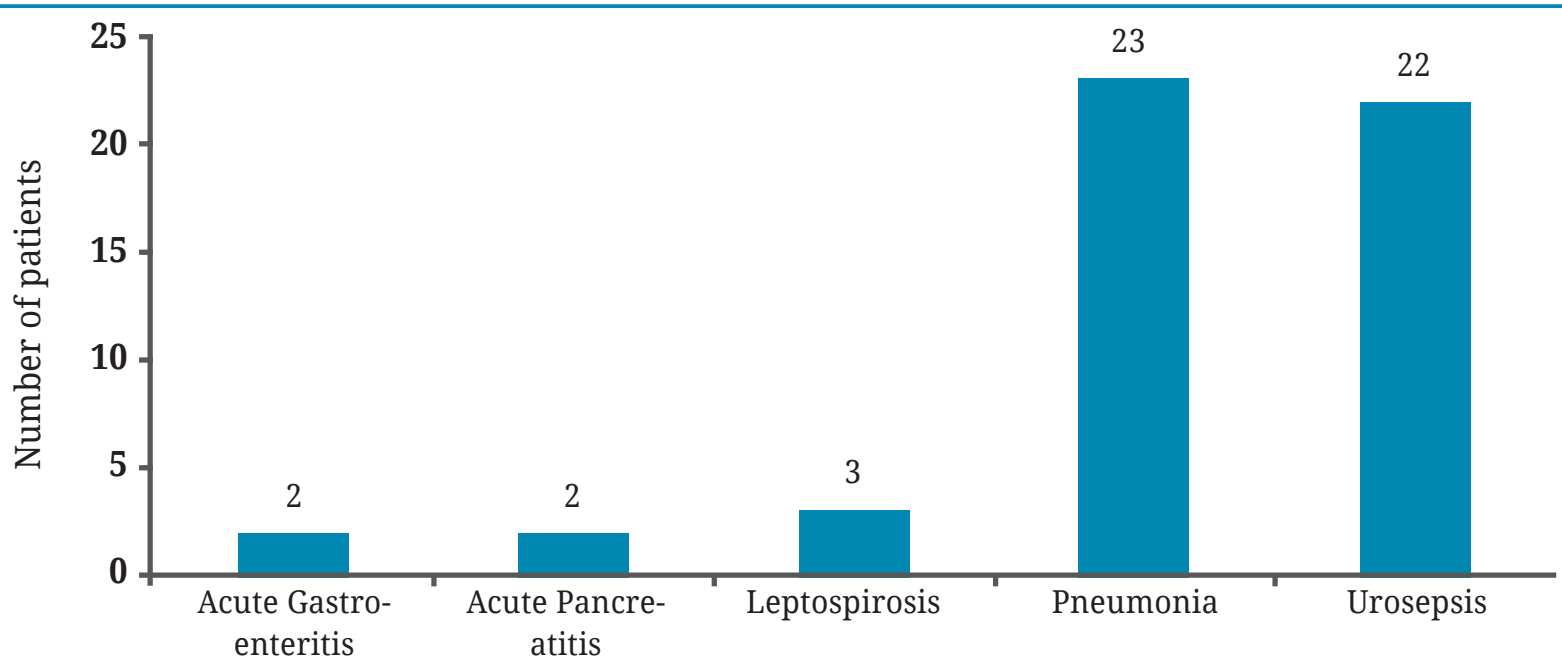

Fig. 3: Number of patients with sepsis and their etiologies $(n=52)$

\section{Table-3: Comparison of risk factors with in-hospital mortality $(\mathrm{n}=69)$}

\section{Characteristics}

Diabetes mellitus

Hypertension

Septic shock on admission

On hemodialysis before admission

On mechanical ventilation

\section{Categories}

No

Yes

No

Yes

No

Yes

No

Yes

No

Yes

\section{No Mortality}

25

25

18

32

24

26

28

22

33

17

\section{In-hospital mortality}

$P$ value

0.24

0.27

0.01

0.25

$<0.01$

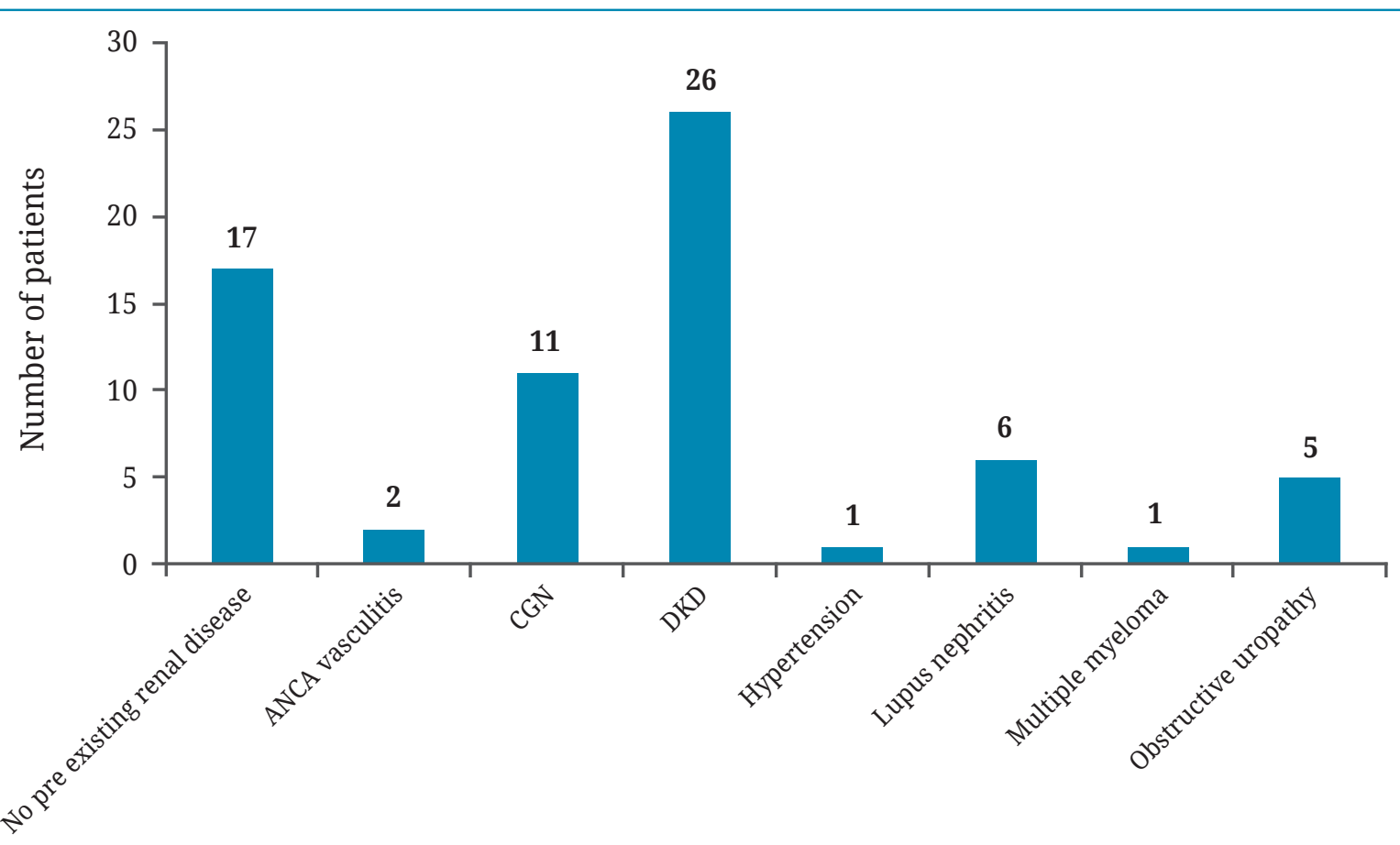

Fig. 4: Pre-existing renal disease $(n=69)$ 


\section{DISCUSSION}

The present study is our experience with SLED at NMCTH. The prevalence of AKI, AKI on CKD ranges from $20 \%$ to $50 \%$ in ICU depending on the definition used. Among this around 30\%-50\% may require some form of RRT. ${ }^{11}$ The current study used the KDIGO definition for AKI and observed that $75 \%$ of patients requiring RRT in ICU were due to AKI alone or AKI on CKD. In 2008 study done by Chhetri et al, ${ }^{12}$ which was designed to look into the prevalence of AKI at the same center found that around $20 \%$ of patients with AKI required dialysis in ICU whereas the current study was designed to look into the RRT in ICU and not the prevalence of AKI.Sepsis was the leading cause of AKI in our study. In a systematic review by Case et al,,$^{13}$ the major cause for AKI requiring RRT in medical ICU was sepsis. In the review, authors defined AKI by AKIN and RIFLE classification, however they did not define sepsis and classified the etiology of sepsis.13 In the current study, pneumonia followed by urosepsis contributed to more than two third of the cases of sepsis. In our study SLED was the modality of RRT in patients on ionotropes which was in difference with study done by Overberger et al, where CRRT were the most common used modality of RRT, with SLED and other "hybrid" treatments used in fewer than $10 \%$ of patients. ${ }^{14}$ This difference of practice may be due to unavailability of CRRT at our center and also CRRT is not a feasible option in resource poor countries like Nepal and moreover SLED could be performed with lower cost, with no anti coagulation and nearly equal solute clearance. ${ }^{15}$ The mean duration of SLED was $14.94 \pm 10.88$ hours in our study which was similar to a Canadian cohort study which compared SLED with CRRT, where the mean SLED and CRRT durations were $14.9 \pm 4.4$ and $15.9 \pm 4.2$ hours/session respectively. ${ }^{16}$ In a major review on RRT in ICU by Ronco et al, the role of RRT was established not only for renal indications but also for other organ-supportive strategies. ${ }^{17}$ In present study, most common indication of RRT in ICU was metabolic acidosis with oliguria which was comparable with a similarly designed study done by Mishra et al. Here the authors defined AKI by the KDIGO classification and used 6 hours session of SLED which was similar to our study. In comparing the serum creatinine among patients who had in hospital mortality versus having no mortality there was no significant difference in our study $(p=0.09)$ whereas in the study conducted by Mishra et al, the serum creatinine was significantly higher in the group of non survivors $(p<0.01)$. This observed difference between the two studies may be due to the mean difference in serum creatinine values in study population and difference in dialysis machinery and protocol. ${ }^{18}$ In a systematic review by Nash et al, ${ }^{19}$ studies on critically ill adults receiving CRRT, HD or SLED to treat AKI were taken. They did not find any definitive advantage for any RRT modality on short-term patient or kidney survival. SLED was suggested as an alternative treatment but still the evidence requires more studies to support this statement. The current study will add on to existing literature in support of SLED.
In the present study, mortality was statistically associated with increasing number of ionotropes (mean 2.05 \pm 1.12 ) and mechanical ventilation implying the severity of disease which was similar to study by Shin et al. ${ }^{20}$ Overall in hospital mortality in patients who received RRT in ICU was less (28\%) as compared to other studies where it ranged from $46 \%$ to $62 \% .{ }^{21,22}$ This difference is due to the difference of inclusion criteria in other studies where they considered only patients who underwent SLED or CRRT. However, our study included all patients who underwent RRT in ICU but the sub group analysis showed statistical significance in mortality when patients had septic shock and/or mechanical ventilation.

There are different names for SLED in the current literature. It is also known as extended daily dialysis (EDD) and prolonged intermittent renal replacement therapy (PIRRT). In SLED, dialysis therapy is extended to 8 to 12 hours with an idea of achieving the hemodynamic benefits of continuous dialysis. In the present study we extended the dialysis therapy upto 6 hours per session which was in accordance with study by Kitchlu et al. However, in their study, blood flow rate and dialysate flow rate was $200 \mathrm{ml} / \mathrm{min}$ and $350 \mathrm{ml} / \mathrm{min}$ respectively whereas in our study it was $150 \mathrm{ml} / \mathrm{min}$ and $300 \mathrm{ml} / \mathrm{min}$ respectively. Further studies are required to answer regarding the optimum duration of therapy in SLED. Currently, SLED is gaining over CRRT due to absence of mortality benefit and higher costs associated with the application of CRRT. Further practical advantages of SLED included the delivery of therapy without anticoagulation (when indicated). ${ }^{16,23}$

The outcome data of our study showed that nearly half of study population could be switched to conventional HD. In a study by Kovacs et al, where they compared the outcome data between SLED and CRRT found no significant differences in terms of renal recovery, days to renal recovery, number of treatments required for each dialysis modality and hemodynamic instability. ${ }^{24}$

Among all the uncertainties regarding the RRT in ICU setting, a recent review published by Bellomo et $a l$ in December 2018 also led to the conclusion that RRT in ICU setting can be provided continuously or intermittently (CRRT or SLED). To date, no modality of RRT has shown superiority over others in terms of mortality and renal recovery. However, multiple observational studies from Canada, France, and Sweden (also disussed above) have identified a link between initial or exclusive use of IHD and decreased likelihood of renal recovery in short term compared with initial or exclusive use of CRRT. Additional studies are required to affirm these observations. ${ }^{25}$ This study has opened up the field of critical care nephrology in Nepal.

Thus in conclusion, SLED can be done in critically ill patients on ionotropes as a hybrid therapy in resource poor setting where CRRT is not available and/or affordable. Moreover, SLED can be performed by conventional dialysis machine with simple adjustments in dialysis parameters and along with existing manpower. 


\section{ACKNOWLEDGEMENTS}

We would like to thank nurses from the ICU and dialysis units for contributing in establishment of SLED service at NMCTH. Sincere thanks to Associate Professor Vinutha Silvanus and Mr. Prem Prasad Pant for helping in statistical analysis.

\section{REFERENCES}

1. Koulouridis I, Price LL, Madias NE, Jaber BL. Hospital-acquired acute kidney injury and hospital readmission: a cohort study. Am J Kidney Dis 2015; 65: 275-82.

2. Rastriya Samachar Samiti. Kidney dialysis is completely free now. The Himalayan Times. Nov 7, 2016; Available from: https://thehimalayantimes. com/kathmandu/kidney-dialysis-completely-freenow/

3. Garg Amit and Parikh CH. Yin and Yang: acute kidney injury and chronic kidney disease. J Am Soc Nephrol 2009; 20: 8-10.

4. Ingelfinger JR, Chawla LS, Eggers PW, Star RA, Kimmel PL. Acute kidney injury and chronic kidney disease as interconnected syndromes. $N$ Engl J Med 2014; 371: 58-66.

5. Deepa C, Muralidhar K. Renal replacement therapy in ICU. J Anaesthesiol Clin Pharmacol 2012; 28: 38696.

6. Greetings from President of Nepal Society of Nephrology (Kafle RK). Available from: http://www. nsn.org.np/about/greetings-from-president.

7. Pannu N, Gibney RT. Renal replacement therapy in the intensive care unit. Ther Clin Risk Manag 2005; 1: 141-50.

8. Acharya SP. Critical care medicine in Nepal: where are we. Int Health 2013; 5: 92-5.

9. Kidney Disease: Improving Global Outcomes (KDIGO) Acute Kidney Injury Work Group. KDIGO clinical practice guideline for acute kidney injury. Kidney Int Suppl 2012; 2: 1-138.

10. Kidney Disease: Improving Global Outcomes (KDIGO) CKD Work Group. KDIGO 2012 clinical practice guidelines for the evaluation and management of chronic kidney disease in adults. Kidney Int Suppl 2013; 3: 1-150.

11. Koeze J, Keus F, Dieperink W, Van Der Horst ICC, Zijlstra JG, Van Meurs M. Incidence, timing and outcome of AKI in critically ill patients varies with the definition used and the addition of urine output criteria. BioMed Central 2017; 18: 1-9.

12. Chhetri PK, Manandhar DN, Pahari LR, Chhetri SS, Chaudhary SK. Acute renal failure in Nepal Medical College Teaching Hospital. Nepal Med Coll J 2008; 10: 132-5.
13. Case J, Khan S, Khalid R, Khan A. Epidemiology of Acute Kidney Injury in the Intensive Care Unit. Crit Care Res Pract 2013; 2013: 1-9.

14. Overberger P, Pesacreta M, Palevsky PM. Management of renal replacement therapy in acute kidney injury: a survey of practitioner prescribing practices. Clin J Am Soc Nephrol 2007; 2: 623-30.

15. Berbece A, Richardson R. Sustained low-efficiency dialysis in the ICU: Cost, anticoagulation, and solute removal. Kidney Int 2006; 70: 963-8.

16. Kitchlu A, Adhikari N, Burns KEA, Friedrich JO, Garg AX, Klein D. Outcomes of sustained low efficiency dialysis versus continuous renal replacement therapy in critically ill adults with acute kidney injury: a cohort study. BMC Nephrol 2015; 16: 1-8.

17. Ronco C, Ricci Z, De Backer D, Kellum JA, Taccone FS, Joannidis M. Renal replacement therapy in acute kidney injury: controversy and consensus. BioMed Central 2015; 19: 1-11.

18. Mishra SB, Singh RK, Baronia AK, Poddar B, Azim A, Gurjar M. Sustained low-efficiency dialysis in septic shock: Hemodynamic tolerability and efficacy. Indian J Crit Care Med 2016; 20: 701-7.

19. Nash DM, Przech S, Wald R, O Reilly D. Systematic review and meta-analysis of renal replacement therapy modalities for acute kidney injury in the intensive care unit. J Crit Care 2017; 41: 138-44.

20. Shin YB, Kim CD, Park SH, Kang HJ, Park JY, Kim YL. Sustained low-efficiency dialysis (SLED) as an alternative therapy to continuous renal replacement therapy (CRRT) in critically ill patients. Korean J Med 2006; 71:163.

21. Marshall MR, Ma T, Galler D, Rankin APN, Williams AB. Sustained low-efficiency daily diafiltration (SLEDD-f) for critically ill patients requiring renal replacement therapy: towards an adequate therapy. Nephrol Dial Transplant. 2004;19:877-884.

22. Ronco C, Bellomo R, Kellum JA. Acute Kidney Injury. Contrib Nephrol. Basel, Karger. 2007;156:320-4.

23. Wald R, Liu K. Critical care nephrology. In: Skorecki K, Chertow GM, Marsden PA, Taal MW, $\mathrm{Yu}$ ASL, eds. Brenner and Rector's the kidney. 10th ed. Philadelphia: Elsevier; 2016. p.21372145.

24. Kovacs B, Sullivan KJ, Hiremath S, Patel RV. Effect of sustained low efficient dialysis versus continuous renal replacement therapy on renal recovery after acute kidney injury in the intensive care unit: A systematic review and meta-analysis. Nephrology. 2017;22(5):343-353.

25. Bellomo R, Wang AY. Renal replacement in the ICU: intermittent hemodialysis, sustained low-efficiency dialysis or continuous renal replacement therapy? Curr Opin Crit Care. 2018;24:437-442. 